\title{
Human Computer Interaction Design for Intensive Care Unit Monitors
}

\author{
$\mathrm{Pu} \mathrm{Liu}{ }^{1,2, *}$ and Yuan $\mathrm{Liu}^{2}$ \\ ${ }^{1}$ Beijing Jiaotong University, No.3 Shangyuan Cun, Haidian District, Beijing, China \\ ${ }^{2}$ Jiangnan University, No.1800 Lihu Avenue, Binhu District, Wuxi, China \\ ${ }^{*}$ Corresponding author
}

\begin{abstract}
Intensive care unit represents the highest level of comprehensive rescuing and nursing of a hospital, and high-tech achievement of medical equipment with strengthened monitoring function supported by computer technologies. Excellent human computer interaction design of the monitors can greatly improve the efficiency and accuracy of medical work. This paper investigates the intensive care unit monitors and tasks, users characteristics, users requirements and operational environment, put forwards design strategies, summarizes design methods, conducts case studies and human factors analysis, discusses the future developmental trend and challenges.
\end{abstract}

Keywords-intensive care unit; human computer interaction; user experience; interaction design; human factors

\section{INTRODUCTION}

Intensive care unit (ICU) is a special department of a hospital, providing intensive medicine treatment integrated with modern medical technologies. The purpose is to offer high quality of medical services to the acute and critical patients who suffer severe and life-threatening illnesses and injuries, namely to conduct physiological monitoring, life support treatment, complication prevention, and to promote and speed up the patient's rehabilitation process.

Human computer interaction (HCI) is a subject that design and evaluate computers for human use. It study the interaction relationship and process between computers and users, and the purpose is to make the communication unobstructed between them. The interaction is a two-way flow of information between people and machine, and the interface is the software or hardware medium that support the interaction.

Along with the development of scientific technology, a variety of monitoring devices are widely used in clinical. In fact, to carry out extensive and close physiological monitoring is the feature and the basis of judgements and treatments of the ICU. Advanced monitoring equipment and monitoring level of the ICU becomes important criteria to measure the level of a hospital.

Meeting the needs of users is the objective of HCI. Good HCI design of ICU monitors facilitate monitoring of physiological parameters of critical patients accurately, and help doctors to treat and cure quickly and effectively. To this end, researching the HCI design of ICU monitors is of great necessity and significance.

\section{ICU MONITORS AND TASK ANALYSIS}

ICUs are equipped with complete medical treatment equipment and monitoring equipment, which monitor and care patients round-the-clock, enabling medical staff to get patients' life information in great quantities and to respond to the information promptly and accurately. ICU monitors are generally divided into ten types according lung, liver, kidney, gastrointestinal tract, blood, metabolism, water-electrolyte, bacteria, cardiovascular and central nervous system. For example, electrocardiograph recording instrument, intravascular and intracranial pressure monitor, hemodynamic and oxygen metabolism monitor, cardiac output computer, pulmonary function monitor, exhaled carbon dioxide analyzer, pulse oximetry, blood gas, blood enzyme, blood biochemistry, and blood glucose monitor, bedside monitor, central monitoring system.

All kinds of vital sign monitors generally consists of the following parts: electrodes or sensors, signal preprocessors, analog signal converters, digital processors and displays. The electrodes and sensors are the basis of monitoring vital signs, directly picking up electrical signals of human bodies or converting the non-electric information of human bodies to electrical signals. Preprocessors amplify and filter electrical signals, in order to improve the signal-to-noise ratio, so as to enhance useful signals and reduce noise interference. Analog signals convert to digital signals, then after digital processing by computer, finally display on the monitor screen. Sound and light alarms are provided when abnormal signals occur.

The equipment or systems monitor the physiological, pathological or biochemical parameters of critical patients continuously, automatically, and real-timely, and realize the automatic analyzing, processing and alarming. They perform the following tasks:

- Monitoring patients' parameters of any changes at any time.

- Identifying and evaluating patients' states.

- $\quad$ Predicting the future states of patients.

- Dealing with emergencies, accidents or potential changes. 


\section{USER CHARACTERISTICS AND REQUIREMENT ANALYSIS}

Medical staff of doctors and nurses, patients and their family members and relatives mainly involve in the ICU.

The doctors' work cover inspection, diagnosis, observation, treatment, rescue, and advice for patients, as well as meeting and discussion. They are equipped with solid medical foundation and rich clinical practical experience. They rise to the emergency, grasp the use of a variety of monitoring instruments and treatment instruments, and analyze clinical monitoring parameters.

Nurses' work includes reading cases, following doctors' advices and orders, taking primary care and treatment, monitoring, recording, assisting emergency rescue, etc. Nurses observe patients, pay close attention to the illness conditions, response promptly and arrive at patients' bedsides in the first time. They master all kinds of nursing technologies, have the ability of judging the changing illness conditions, and correctly use all sorts of monitors and accurately record data.

Support staff includes laboratory technicians, radiation technicians, engineers and other technicists, who assist doctors to complete the work of the diagnosis or maintain the machines.

Therefore, the HCI design of ICU monitors should firstly meet the requirement of activities of care, treatment, and rescue. Usability demand of convenience and efficiency is the basic requirement to reduce intensely heavy workload. Medical staff of ICU work for a long time in a closed unit repetitively and tensely, and face patients of acute and severe stage. They work under great psychological pressure. So providing them with pleasure equipment environment becomes the urgent demand of user experience.

The service object of ICU are critically ill patients who come from various clinical departments and suffer serious insufficiency of breathing, circulation, metabolism, or acute malfunction of some important viscera. They are life threatening at any time. Because each patient individual varies in the aspect of illness state, severe degree, age, gender, growth status, education background, living habits, etc., their demands will be different. Majority of patients of ICU are without ambulation ability and keep in bed and indoor. Their basic physiological needs are to rely on medical staff and facilities, and on the monitoring, care and management within comfortable and pleasant environment. The basic psychological needs includes privacy, to be respected, and to communicate with others.

Family members and relatives refers to those people whose daily lives is closely associated with the patients, whose lives will be affected seriously when the patients are in the ICU. Recognizing their roles in the treatment and nursing, and providing rational information about the patients, can facilitate communication between medical staff and the families, and help to build family stress and adaptation mechanisms. All of those are beneficial to patients with psychosomatic rehabilitation.

\section{OPERATIONAL ENVIRONMENT}

ICUs' integrated environment are different from common wards. They must be fitting for rescuing and prevent infection.

ICUs maintain air cleanliness level 10000 , relative humidity $50 \% \sim 60 \%$, indoor temperature $24 \pm 1.5^{\circ} \mathrm{C}$, set up independent air conditioning control system, use the laminar flow purification devices to prevent pollution and cross infection.

In order to take first aid and transfer, ICUs must ensure sufficient power supply, so they are equipped with double power and emergency power. Important instruments are guaranteed by uninterruptible power supply and leakage protection settings. Each bed has independent feedback circuit and multiple power sockets and wires, considering a variety of devices used at the same time when rescuing.

Each unit is equipped with comprehensive medical hoist tower, providing electricity, oxygen, compressed air and vacuum suction support, as well as a variety of gas pipeline and transfusion pumps.

Ceilings are seamless or sealed for all kinds of instruments fixed. Ceilings, walls, beds, boards, and various devices' surfaces are resistant to corrosion, pollution and easy to clean. Doors and windows are of airtightness in order to reduce the air vibration.

\section{Strategy and Method of HCi Design for ICU MONITORS}

After investigating human, machine and environment, the task of the design mixes with technology, aesthetics, and humanity considerations. There is no way fully reflect the complexity of all design factors. Generally speaking, the typical method of user centered design goes throughout the whole process of the design. The assessment and evaluation serve to improve the design. The user centered design method turns the goal to human, setting users to be the core part of design and development. It analyzes the, perception, cognition and information processing mode of users, and emphasizing on user requirements. It has become the mainstream of today's industry circle.

There are some basic principles must be followed when design for ICU monitors. Alan Cooper and Donald Norman lay the theoretical foundations. Ben Shneiderman's eight golden rules, Jakob Nielsen's ten usability heuristics provide standards for human computer interaction design and evaluation.

In addition, $7 \pm 2$ rule, 2 seconds rule, 3 clicks rule, Pareto's law, Fitt's law, John Maeda's 0123 law and the Laws of Simplicity, Bauhaus design concept of form follows function, Skeumorphic design concept of Apple company are all important reference rules when design for ICU monitors.

Beyond that, due to the special feature of ICU, the design must emphasize the following considerations:

\section{A. Patient-Oriented}

It is the first principle of ICU treating and nursing work, reflecting the hospital purposes of serving patients. ICU patients suffer physical pain caused by diseases or injuries. 
They also experience extremely psychological complexity in the process of treatment. The monitors and systems shall put the patients' healing as the starting point and end-result.

\section{B. Security}

Hospital is a place to heal the wounded and rescue the dying. In ICUs, the doctors and nurses carry out treatment and nursing work based on monitoring information, which is closely related to life safety of patients. The patients' conditions change quickly. Monitoring technologies are complicated but the most important part is to ensure information security.

\section{Preventability}

It is valuable to carry through preventive monitoring in each steps of the process, so as to make all kinds of unsafe factors under control, so as to ensure the safety of the patients. When making design schemes, it is better to think possible problems, specify key points and develop prevention methods, so mistakes or accidents can be noticed and observed in time, and dangers nip in the bud.

\section{Efficiency}

Monitors are used in the busy clinical situation. Doctors or nurses need real-time monitoring information of patients. They have strict demands on effectiveness and efficiency. The practicability, operability, learnability are all should be counted in when designing. Friendly and optimized interactive interface contributes to simple, convenient and efficient operation.

\section{E. Data-Based}

There are a large number of physiological data and information in clinical monitoring of ICU. The interaction and interface design should emphasize effective data collecting and displaying. The objective data exactly reflect the patients' actual conditions, rely on which patients can be effectively diagnosed.

\section{F. Extensibility}

Separate the function module structure and user interface definition, so the monitors can be customized according to the requirements of various users. Consider extensibility of the monitors or systems, so that the clinical information sharing, real-time reading and mutual using will be easier. Clinical data collecting also can be facilitated through extending various wireless devices.

\section{G. Entirely-Integrated}

As the whole process of care and treatment involves medical staff, patients, relatives, devices and institutions. The design should not neglect the whole system. All elements of the incorporated system are to be considered including people, technology, equipment, medication, environment and time, etc. Make sure the design integrated in ICU environment and emergency scenario, and the hospital information management systems.
The purpose is to serve people. When the basic function needs are met, designers should care about users' emotional and spiritual comfort. Psychologist Abraham Harold Maslow divides human needs into five levels. People turn to higher ones when the base ones are met. Emotional design devote to meet psychological demand and get users' emotional recognition. It can ease people's fears of high-tech equipment. Making sure the usability of equipment is one aspect, and studying the users experience and feelings is the other important aspects. It has a vital influence on the design. Through the elaborate collocation of material, shape, colour, light, sound, interactive effects, and the reasonable function configuration, the concerns for users can be embodied.

Medical staff of the ICU are long-term users. They observe monitoring screen closely and frequently, and timely response to the changing illness conditions. The high-intensity work for a long time makes them high fatigue of body and mind. At the same time they have to face the sickness and death of critically ill patient. All these lead to psychological pressures. Designers should pay more attention to their needs. It is urgent to solve the current problem of overload information of monitoring systems, and high false alarm rate as much as possible, providing simple, clear and definite information to assist their decision and diagnosis. It is also important to design various functional details according human engineering theories, and as far as possible to consider the use convenience and set a comfort working environment which have not too much interference and is helpful to relieve the pressures.

Patients confront cold devices, wires and tubes in their tough time. It is easy to be susceptible to helplessness and isolation emotions. Surrounded by strange sounds and activities 24 hours in the process of their consciousness gradually restoring, patients may hear monitor alarming, the ventilator and other equipment operating, strangers' talks, etc. With the oral intubations or oxygen masks they cannot word. They cannot communicate with medical staff about their concerns of the surgery effect, fears of the strange environment, discomforts and inconveniences of the limb protective constraint. At this point, the monitoring equipment of the ICU aggravates the patients' experiences of fear, anxiety and nervousness. The monitor alarm sounds arrive a maximum value of 80 decibels, seriously handicapping the patient's rest and sleep, thus affect the patients' recovery. So the HCI designers of ICU monitors must consider the colors, lights and sounds, what are the physical and psychological influences to the patients, and how to meet patients' needs and to build a warm medical environment and reduce the patients' undesirable moods.

\section{CASE StUdy and Human Factors Analysis}

Excellent HCI design of ICU monitoring equipment are all of great user experience with vivid and clear user interfaces that bring easy and safe operations to doctors, nurses, physiotherapists and technicians, and with thoughtful considerations of human factors that bring consolation to patients and their families.

The Advanced NICU Hybrid by Philips is the specially designed ICU ward for babies born prematurely or with 
complications. Compared with the other intense and public ICU environment, this ward tightens the bond of babies and their mothers, and avoids negative impact on their neurological developmental outcomes in the intensive and critical time. It is a whole room of clean and comfortable internal environment with basic living facilities of bed and entertainment, suitable for families of premature babies to relieve stress and promote parental bonding and involvement in the difficult time. The Design protects infant sleep and reduces negative stimuli to improve the quality of life.

The display screens of monitors are embedded on the walls and cabinets, monitoring babies' circulation, respiration, metabolism or infection information and the ward environment. They are located at higher places, so they are easy to observe, at the same time avoid the interference of other electronic instruments or interruption of babies sleeping. Abandoning the traditional HCI design of monitors, there are no wires or tubes. The screens like children's blackboards, cooperating with walls of overall tonal environment of high lightness, low color purity and wooden surface, it brings relaxed, warm and clean feelings and helps the babies gets good rest. There are also colorful photographs, drawings, and toys below the screens. The monitoring equipment environment is fit for babies' psychological characteristics and meet their families' needs.

SERVO-u and SERVO-n Ventilators by Swedish company Veryday is an easy-to-use breathing machine of intensive care and emergency medical products. Due to takes into account the user experience innovatively, the crucial life-saving equipment is designed to be used in a more secure and easy way. It makes the complex operations easier. Veryday design team researches the gap between the user's knowledge and the capacity of the breathing machine. They focus on narrowing the gap. The touch screen interfaces are provided to users with image preview, built-in guide and automatic calculation.

There are three display screens of monitoring. For the medical staff and remote observation, the screen views give sufficient information of patients' physical signs like traditional mode. However, on patient's bedside, the screen shows relieved background, limited information, and with no alarm. It do not distract patients and their visitors. The simple and intuitive interface improve user experience and life quality of the patients.

The monitoring display screen adopts a natural background picture of blue sky, green grass, sunshine and dew. A big yellowish-green ladybird pattern is embedded on the main body of the machine. In the green environment, human skin temperature reduce about $2^{\circ} \mathrm{C}$, the heartbeat reduce $4 \sim 8$ times per minute, and the breath slow down. Patients who long time stay in ICUs are unable to breathe the fresh air and their bodies lack activities, so they eager to get close to the nature. This design introduce natural elements into ICU equipment, making patients mentally and physically relaxed.

\section{PROSPECT AND DISCUSSION}

The ICU is of characteristics of highly comprehensive technology. Internet, mobile Internet, big data and cloud computing and new generation information technology rapidly develop and actively permeate into medical technology including ICU application. With rapid progress of genome sequencing technology, 3D organ printing, wearable devices, robotic surgery, cross application of biological information and big data, information integrating and sharing and other related technology, the future ICU monitoring equipment and environment will optimize diagnosis and treatment quality, and provide more accurate service mode. The HCI design will have new breakthrough and development in this field.

Along with the influx of more complex content and data, and the involvement of a variety of devices with different interactive dimensions, the design and iteration process continuously changes. Designers, experts, engineer, planners with different specialties collaboratively work together as a whole to achieve the goal. It is a systematic field. The challenge of user experience design of HCI is how to minimize interaction as far as possible, and to really focus on the user's behavior, rather than to have the complicated instruments become more confusing. Technology should be as low as possible to attract the attention of their users, while create seamless, ubiquitous user experience. The goal is invariable, that is to create a comfortable healing environment for patients to alleviate their tension, anxiety and other negative emotions, to provide medical staff an efficient working environment at the same time relieve their pressure of high-intensity work. Do the utmost to care needs and feelings of every individual.

\section{ACKNOWLEDGMENT}

Jiangnan university independent scientific research program youth fund JUSRP11556.

\section{REFERENCES}

[1] Agarwal S, Pandey G N. Human computer interface design for neonatal intensive care with data mining[C]// International Conference on Intelligent Human Computer Interaction. 2012:1-6.

[2] Michel A, Benson M, Junger A, et al. Design principles of a clinical information system for intensive care units (ICUData).[J]. Studies in Health Technology \& Informatics, 2000, 77:921.

[3] Norman D A. Human-centered design considered harmful[J]. Interactions, 2005, 12(4):14-19.

[4] Effken J A, Loeb R G, Kang Y, et al. Clinical information displays to improve ICU outcomes.[J]. International Journal of Medical Informatics, 2008, 77(11):765.

[5] Staggers N. Human factors: imperative concepts for information systems in critical care.[J]. Aacn Clinical Issues Advanced Practice in Acute \& Critical Care, 2003, 14(14):310-9; quiz 397-8.

[6] Koch S H, Staggers N, Weir C, et al. Integrated Information Displays for ICU Nurses: Field Observations, Display Design, and Display Evaluation[J]. Human Factors \& Ergonomics Society Annual Meeting Proceedings, 2010, 54(12):932-936.

[7] Marcus A. Principles of Effective Visual Communication for Graphical User Interface Design[J]. Readings in Human-computer Interaction, 1995, 19(2):425-441.

[8] Bhutkar G, Katre D, Deshmukh S. Overview of Critical Factors Affecting Medical User Interfaces in Intensive Care Unit (ICU)[J]. Health, 2013, 2(4):33-45. 